\title{
LAS RELACIONES ENTRE EL SIEE, PEI Y LA EVALUACIÓN EN EL AULA: ANÁLISIS DE CASO EN LA INSTITUCIÓN EDUCATIVA OLIVERIO LARA BORRERO DE LA CIUDAD DE NEIVA.
}

Claudia Mercedes Trujillo Villegas*

\section{INTRODUCCIÓN}

La educación juega un papel protagónico en el desarrollo del país y su calidad tiene estrecha relación con la evaluación. Una de las competencias básicas que un docente debe desarrollar es evaluar en su verdadero sentido, y esto es lo que hace que el docente sea formador; que apunte al desarrollo integral de los estudiantes y deje de ser un instructor. Un docente así, es una pieza fundamental de la calidad educativa en el aula y día a día busca el mejoramiento y el perfeccionamiento de sus alumnos.

La diversidad de concepciones o perspectivas que se han dado desde el siglo XX en el campo de la evaluación presenta también las diferentes relaciones que el docente ha establecido con la enseñanza, el aprendizaje y en general con las practicas pedagógicas.

En Colombia, la evaluación ha empezado a ser concebida como un componente pedagógico esencial en el proceso de enseñanza aprendizaje y se espera que las instituciones educativas entiendan que la evaluación pedagógica entraña elementos más formativos que académicos en dicho proceso, desde sus Sistemas Institucionales de Evaluación.

Determinar las posibles dimensiones de coherencia entre el Sistema de Evaluación de Estudiantes SIEE y la evaluación en el aula en los grados y áreas seleccionados, permitirá, también desarrollar en la institución cuerpos académicos que permitan proporcionar rutas posibles para identificar algunos problemas y trabajar colectivamente y así, determinar cuáles serían los presupuestos de calidad frente a la evaluación en el contexto de la Institución Educativa.

Esta investigación busca analizar el proceso de evaluación tal y como lo concibe la institución, los docentes y el sistema de evaluación e identificar, las diferentes reflexiones, concepciones y racionalidades que influyen directamente en las intencionalidades y finalidades de las prácticas evaluativas. Con el análisis también se busca re-significar procesos evaluativos institucionales, lo cual implica concebir la evaluación como herramienta dinamizadora de los procesos educativos, que contribuya a potenciar las capacidades del ser humano, favoreciendo un desarrollo integral de los sujetos y al mismo tiempo a dinamizar los procesos institucionales.

Las propuestas están centradas en la conformación de un equipo de gestión académica interdisciplinaria, que permita desarrollar cuerpos colegiados encaminados a diseñar e implementar rutas evaluativas académicas coherentes y, así mismo, el monitoreo permanente de las estrategias e instrumentos de evaluación, que estén encaminados a articular las concepciones teóricas de evaluación y sus prácticas. 


\section{RESUMEN}

Los objetivos de este proyecto se orientan a analizar el SIEE y las prácticas de evaluación asociadas a su constitución, así como, a determinar las concepciones de coherencia que tienen los docentes y directivos frente a la evaluación y sus alcances en la evaluación en el aula; el estudio se realizó en la Institución Educativa Oliverio Lara Borrero, desde un modelo cualitativo, cuyo enfoque fue el análisis de casos el cual permitió al investigador adentrarse en el proceso de evaluación como lo concibe la institución, los docentes y el sistema de evaluación. Los resultados arrojados por este trabajo muestran que los docentes y directivos, en lo que a las concepciones de evaluación se refiere, presentan un razonamiento formativo y un acercamiento a la definición de la evaluación de la enseñanza para la compresión pero, se pierde cuando, la planeación de aula, las estrategias, instrumentos y ejecución se convierten en un proceso de racionalidad sumativa; de este modo, aunque se hable de autoevaluación y retroalimentación finalmente el resultado es numérico para responder a los propósitos de la promoción según el acuerdo 04. El equipo de docentes y directivos está por construir relaciones de coherencia entre el SIEE, el PEI y la evaluación en el aula de manera explícita, pues se logra evidenciar en esta investigación que existe una clara contradicción conceptual; frente a sus discursos, el modelo pedagógico de la institución y sus prácticas pedagógicas. No hay duda sobre los grandes esfuerzos que los maestros y directivos participantes de este estudio realizan para ayudar a los jóvenes a aprender. Sin embargo no es clara la relación entre dichos esfuerzos y las evaluaciones propuestas en el aula. No se encuentra correspondencia didáctica entre los resultados de las evaluaciones y las estrategias de la enseñanza para promover los cambios necesarios para superar dificultades. Es imperante, ante la ausencia de liderazgo académico entre los directivos, enlazar la gestión con lo académico para generar procesos pedagógicos innovadores, reflexivos y corresponsables frente al proceso evaluativo.

La ruta que se propone, entonces, es valorar de forma integral a los estudiantes lo cual evita ver la evaluación como una práctica limitada a resultados finales; es necesario articular el currículo con los contextos, de tal manera que haya pertinencia de los conocimientos que son objeto del aprendizaje. La evaluación debe ser una práctica reflexiva que debe propender por la comprensión y mejora de los procesos de enseñanza y aprendizaje.

PALABRAS CLAVES Evaluación Formativa, Evaluación de Progreso, Reflexión Pedagógica de la Evaluación

\section{OBJETIVO GENERAL}

Determinar las posibles dimensiones de coherencia entre el Sistema de Evaluación de Estudiantes SIEE y la evaluación en el aula en los grados tercero, quinto, noveno y once en las áreas de Lenguaje, Matemáticas y Ciencias Naturales.

\subsection{Objetivos Específicos}

a. Analizar el SIEE y las prácticas de evaluación asociadas a su constitución.

b. Determinar el concepto de coherencia que tienen los directivos y docentes frente a la evaluación y sus alcances en la evaluación en el aula.

c. Analizar posibles rutas evaluativas que estén en conjunción con el SIEE y el PEI Institucional. 


\section{METODOLOGÍA}

\subsection{Descripción del Estudio}

La investigación con enfoque de estudio de casos pertenece al paradigma cualitativo interpretativo. El paradigma cualitativo interpretativo considera que la realidad es construida por las personas involucradas en la situación que se estudia; y aunque las versiones de los mundos sean personales, estima que las construcciones no son completamente ajenas, sino que se parecen, tienen puntos en común. Así, las realidades construidas por personas distintas pueden tener puntos de coincidencia, ser compatibles (Stake, 1995).

Estas múltiples realidades necesitan ser reportadas; ya sea con ejemplos de los diálogos de los informantes, presentando temas que reflejen sus palabras desde diferentes perspectivas. El propósito de la investigación no es descubrir la realidad, sino construir una cada vez más clara y sólida, que pudiese responder a la duda sistemática ( Stake, 1995).

\section{DESARROLLO Y RESULTADOS}

\subsection{Análisis Documental}

A continuación se presenta el balance documental que se obtuvo luego de la lectura minuciosa de los diferentes documentos de tipo legal como Decretos reglamentarios, Resolución del SIEE, PEI y Actas de las comisiones de Evaluación y promoción de la Institución. Se obtuvieron unos criterios para el análisis con el fin de visibilizar las tendencias: ¿Identificar el concepto de evaluación?, ¿Cuál es el objetivo final de la evaluación?, ¿Cuáles son las prácticas evaluativas asociadas a su constitución?.

Estas preguntas fueron el eje de análisis y con base en ellos está estructurado el balance $y$, posteriormente se plantean conclusiones generales. A continuación se presenta una descripción general de los documentos objeto de este balance documental:

\begin{tabular}{|l|l|l|}
\hline \multicolumn{1}{|c|}{ DOCUMENTOS } & \multicolumn{1}{|c|}{ DESCRIPCIÓN } & TIPO DE DOCUMENTO \\
\hline $\begin{array}{l}\text { PEI } \\
\text { Proyecto Educativo } \\
\text { Institucional }\end{array}$ & $\begin{array}{l}\text { Decreto 1860/94, artículo 14. Toda } \\
\text { institución educativa debe elaborar y } \\
\text { poner en práctica con la participación } \\
\text { de la comunidad un proyecto } \\
\text { educativo que exprese la forma como } \\
\text { se ha decidido alcanzar los fines de la } \\
\text { educación definidos por la Ley } 115 \text { de } \\
1994 .\end{array}$ & $\begin{array}{l}\text { Libro reglamentario } \\
\text { Carta de navegación } \\
\text { institucional }\end{array}$ \\
\hline $\begin{array}{l}\text { Decreto 1290,16 de abril } \\
\text { de 2009. }\end{array}$ & $\begin{array}{l}\text { El decreto reglamenta la evaluación } \\
\text { del aprendizaje y promoción de los } \\
\text { estudiantes de los niveles de } \\
\text { educación básica y media que deben } \\
\text { realizar los establecimientos } \\
\text { educativos. }\end{array}$ & $\begin{array}{l}\text { Decreto reglamentario de } \\
\text { evaluación }\end{array}$ \\
\hline $\begin{array}{l}\text { SIEE } \\
\text { Sistema Institucional de } \\
\text { Evaluación de los } \\
\text { estudiantes }\end{array}$ & $\begin{array}{l}\text { Decreto 1290/2009, artículo 8, } \\
\text { creación del sistema institucional de } \\
\text { evaluación de los estudiantes. }\end{array}$ & $\begin{array}{l}\text { Acuerdo 04 de Diciembre } \\
\text { de 2012 }\end{array}$ \\
\hline $\begin{array}{l}\text { Actas de Comisión de } \\
\text { Evaluación y promoción }\end{array}$ & $\begin{array}{l}\text { Libro de actas de evaluación y } \\
\text { promoción, se diligencian en forma } \\
\text { consecutiva por cada nivel y grado en } \\
\text { cada periodo académico y al finalizar } \\
\text { el año escolar. }\end{array}$ & $\begin{array}{l}\text { Libro de actas institucional } \\
\text { de las comisiones de } \\
\text { evaluación y promoción del } \\
\text { año lectivo. }\end{array}$ \\
\hline
\end{tabular}




\section{Concepto de evaluación: Tendencias generales}

En el PEI institucional, en el capítulo: Componente Pedagógico, define la evaluación como un proceso permanente, cualitativo, dinámico y sistemático. Esta evaluación permitirá verificar el alcance de los logros y reorientar procesos para mejorar la calidad educativa. El decreto 1290, en el artículo 1, delimita la evaluación en tres ámbitos: Internacional, Nacional e institucional. En cuanto al ámbito institucional, el decreto, en el artículo 3, define los propósitos de la evaluación institucional de los estudiantes y enumera cinco propósitos: Identificar las características personales, interés, ritmos y estilo de aprendizajes de los estudiantes para así valorar sus avances.

1. Proporcionar información para consolidar o reorientar procesos educativos relacionados con el desarrollo integral.

2. Suministrar información para implementar estrategias pedagógicas para apoyar desempeños superiores o estudiantes con desempeños bajos.

3. Determinar la promoción de los estudiantes

4. Aportar información para el ajuste al plan de mejoramiento institucional.

En cuanto al SIEE institucional, llamado acuerdo 04, ver Anexo A, en el artículo 4, define la evaluación como un sistema tal como lo establece para todo el país el MEN a través de la Ley General de Educación y demás normas concordantes entre ellos el reciente Documento No.11, Finalidades y alcances del decreto hace mención a:

A la caracterización integral, formativa, motivadora, pertinente, orientadora, pero nunca sancionatoria; utilizando las diferentes técnicas de evaluación y haciendo triangulación de la información, para emitir juicios y valoraciones contextualizadas; así mismo una evaluación incluyente y de respeto por la diferencia, centrada en la forma como el estudiante aprende, sin descuidar la calidad de lo que aprende; que sea transparente, continua y procesual y convocando de manera responsable a todas las partes en un sentido democrático y aplicando la autoevaluación integral en ellas. Las comisiones de evaluación y promoción que funcionan al finalizar cada periodo académico y conformado por los coordinadores y docentes de cada nivel y grado, toman los resultados arrojados por el software de notas, que especifica en cada grado y a cada estudiante el número de áreas con su correspondiente valoración, la comisión retoma los criterios establecidos en el SIEE, artículo 5, pero, específicamente lo que tiene que ver las áreas y sus valoraciones para determinar cuántas áreas aprobadas o reprobadas presentan. Para efectos de evaluación y promoción y en su autonomía la comisión determina mediante acta quienes son promocionados, reprobados o quienes pueden solicitar la promoción anticipada.

\section{Objetivo final de la evaluación: Tendencias generales}

EI PEI, plantea la evaluación como objetivo, verificar el alcance y reorientar los procesos para mejorar la calidad educativa; este objetivo coincide con lo planteado en el decreto 1290, artículo 3, en cuanto a, que, la evaluación permitirá orientar procesos educativos relacionados con la formación integral del estudiante. EI SIEE reglamenta la evaluación y promoción de los estudiantes, en el artículo 5: Criterios de Promoción y evaluación, de igual manera las comisiones de evaluación y promoción al finalizar el periodo, de acuerdo al consolidado arrojado ejecutan lo reglamentado en el SIEE. Prácticas de evaluación asociadas a su constitución: Tendencias generales Dentro de las estrategias y prácticas evaluativas el PEI, en el componente pedagógico menciona que de acuerdo al modelo pedagógico de la institución la estrategias de evaluación están encaminadas a 
tener un enfoque constructivista en donde los aprendizajes a evaluar serán colaborativos, autónomos que buscan valorar el desempeño y la promoción humana, al igual las pruebas ICFES-SABER.

El decreto 1290 en el artículo 4, Definición del sistema institucional de estudiantes, lo incorpora al PEI; pero también en el artículo 6 y 7, establece la promoción escolar y los lineamientos que la institución educativa debe insertar en el SIEE. Lo que permite que a partir de estos artículos las instituciones educativas en su autonomía determine el proceso a seguir. En el caso del SIEE, acuerdo 04, el artículo 8, 9 y 10, reglamenta cuales son las estrategias de valoración integral, las acciones de seguimientos y los procesos de autoevaluación de los estudiantes. En cuanto a las comisiones de evaluación y promoción nuevamente de acuerdo a los resultados finales valorativos determinan su promoción.

De lo anterior se puede concluir que:

- Luego del análisis anterior se pueden identificar tres tendencias generales que permiten visibilizar la evaluación a partir de lo conceptual, pedagógico y normativo. En los documentos analizados: PEI, decreto 1290, SIEE y las Comisiones de Evaluación y promoción. La primera, sobre el concepto de evaluación. La segunda tendencia con el objetivo primordial de la evaluación y la tercera respecto a las prácticas y estrategias evaluativas.

- Respecto al concepto y objetivo de la evaluación se puede concluir que existe algunas relaciones de coherencia respecto a lo que concibe la institución y los docentes investigados frente a las instancias pedagógicas PEI, Decreto 1290 y SIEE, en cuanto a definir la evaluación como integral, flexible, pertinente y orientadora e incluyente, que permita identificar lo que el estudiante aprende. En cuanto a las comisiones de evaluación y promoción, se evidencian que cumplen la labor de sistematizar, realizar un balance porcentual y numérico frente a las valoraciones, para efectos de promocionar un estudiante, según lo reglamentado en el acuerdo 04, SIEE. No es claro identificar al interior de las Comisiones de Evaluación y Promoción, ya que estas solo cumplen la labor normativa y de ejecución mediante acta la decisión de promocionar a los estudiantes.

- Las estrategias y prácticas evaluativas en el PEI, se identifican en el modelo pedagógico y están encaminadas a estrategias colaborativas y constructivistas. El decreto 1290, permite a través de los artículos 6 y 7 establecer los criterios y lineamientos de promoción, que deben insertarse en el SIEE. De esta manera se puede identificar que tanto el PEI, decreto 1290, desencadenan una serie de acciones conceptuales, pedagógicas para ser desarrolladas y ejecutadas mediante el SIEE y las comisiones de evaluación y promoción.

- Finalmente se puede concluir que, respecto a los elementos esenciales en la construcción de un sistema institucional de evaluación y sus dinámicas en el proyecto educativo, y respecto a los procesos de legitimidad, es de gran importancia mantener las comisiones de evaluación y promoción, pero; estas comisiones deben apoyarse en las decisiones de los equipos pedagógicos y no limitarse simplemente a cumplir taxativamente una labor normativa en donde solo prime porcentajes y valoraciones numéricas. De esta manera es importante no olvidar la perspectiva social e integral de la evaluación y la actitud pedagógica asociada a la evaluación. 


\section{CONCLUSIONES}

A continuación, se desarrolló un análisis que consiste en realizar un contraste de información a partir de las entrevistas y las encuestas trabajadas en tres dimensiones:

a. Concepto de evaluación pedagógica: Dentro esta dimensión se analizan las concepciones evaluativas de los docentes y directivos.

Los docentes y directivos conciben la evaluación pedagógica como un proceso integral que permite la apropiación del conocimiento a través del seguimiento e implementación de estrategias de retroalimentación y además la considera como sinónimo para determinar el avance de los logros de los estudiantes, así mismo considera como finalidad medir y verificar el proceso con el ánimo de identificar fortalezas y fallas en el proceso, consideran que la evaluación debe ser conceptual, continua permanente y flexible.

De lo anterior se puede inferir que los docentes y directivos en cuanto al concepto de evaluación presentan un razonamiento formativo, presentan un acercamiento a la definición de la evaluación de la enseñanza para la compresión, como un proceso que brinda respuestas claras a los desempeños de comprensión de los estudiantes de tal manera que permita mejorar sus desempeños pero, no se puede evidenciar en esta investigación como sus dos componentes principales: La valoración y la retroalimentación, no proceden a explicar cómo ha alcanzado el proceso de la enseñanza y los aprendizajes posteriores y se limita a expresarlo en un valor numérico.

b. Relaciones PEI, Modelo pedagógico, SIEE: Esta dimensión analiza el sentido y las relaciones de coherencia de la evaluación en los tres componentes.

En cuanto las relaciones del PEI, el modelo pedagógico, SIEE y la evaluación en el aula apuntan a describir la evaluación como continua, integral y flexible e identifican el modelo pedagógico institucional, en cuanto al SIEE, acuerdo 04 mencionan algunas estrategias e instrumentos y en particular el proceso de promoción pero, no hay claridad explicita sobre esas relaciones, los docentes no logran determinarlas. No se evidencia una correspondencia didáctica entre los resultados de las evaluaciones y las estrategias que promuevan y permitan superar dificultades.

c. La evaluación en el aula: En esta dimensión se analiza las estrategias, planeación, instrumentos y ejecución de la evaluación.

Dentro del proceso de planeación y ejecución de la evaluación se evidencia en los docentes una concepción enciclopedista, los docentes son los que determinan cuales son los contenidos a trabajar en cada nivel y área así como los indicadores de desempeños que deben cumplir, los instrumentos de evaluación son variados que apuntan a determinar desempeños grupales e individuales.

De la información anterior se deduce que las estrategias más sobresalientes dentro del proceso de formación y evaluación de los estudiantes se encuentra la prueba tipo SABER, los trabajos escritos, la consulta, la evaluación oral, en cada una de ellas se desarrollan de manera grupal, y las evaluaciones escritas tipo prueba SABER se hacen de forma individual, presentando notables diferencias en los resultados ya que las valoraciones son bajas. Situación que se evidencia aun más en la evaluación acumulativa que se realiza cada semestre y que es utilizada como estrategia de superación y promoción.

1 TYLER, R DIVERSAS CONCEPCIONES SOBRE EVALUACIÓN. En

http://wWw.monografias.com/trabajos69/evaluacion-educativa-planteamientos- teóricos -básicos/ evaluación- educativa-planteamientos- teóricos- basicos2. 
La autoevaluación y la evaluación de procesos actitudinales se presentan acorde a los planteamientos de Tyler que expresa que "el propósito de la evaluación es esencialmente el de determinar en qué medida los objetivos educacionales son realmente conseguidos por el programa, plan o proyecto propuesto". Dado que los objetivos educacionales tienden a señalar los cambios que se van a producir en la conducta del educando, entonces la evaluación es el proceso para determinar el grado en que se están verificando estos cambios de comportamiento"1.

La retroalimentación debe demostrar el resultado de los desempeños y la posibilidad de mejorarlos, en la planeación de las clases y las actividades. De esta manera el proceso evaluativo debe responder a una práctica reflexiva que lleve a la mejora de las prácticas pedagógicas.

En lo anterior podemos inferir que el concepto de evaluación pedagógica formativa con una clara influencia de la enseñanza para la comprensión, se pierde cuando en el aula en la planeación, las estrategias, instrumentos y ejecución se convierte en un proceso de racionalidad sumativa, porque aunque se hable de autoevaluación y retroalimentación finalmente el resultado es numérico al finalizar los periodos, para responder a los propósitos de la promoción según el acuerdo 04.

La racionalidad formativa en este caso estaría orientada a comprender que la retroalimentación debe ser un proceso constante dentro de cualquier evaluación, tendiente a observar el proceso de construcción de conocimiento. La retroalimentación es considerada como un proceso de diálogo entre el profesor y el estudiante, en el cual se analizan los aspectos bien hechos en una evaluación, y los que se deben mejorar, esta mejora no solo implica indicar lo que esta errado, sino que debe existir un trabajo a partir del error, en el cuál se analicen las causas de éste y se busquen junto al estudiante los caminos para mejorar ir orientando al estudiante en dicha construcción.

En cuanto a los propósitos de la evaluación que plantea el decreto 1290, en el artículo 3; sobre las características personas, interés, ritmos de desarrollo y estilos de aprendizaje. EI SIEE no lo desarrolla y se evidencia una clara contradicción con los desempeños que en lugar de reconocer diferencias, las desconoce por su uniformidad.

Finalmente los resultados obtenidos en la aplicación de los diferentes instrumentos permiten evidenciar una debilidad en el discurso que manejan los docentes referente al concepto de evaluación, discurso que evidencia una inclinación hacia la evaluación formativa pero, la evaluación no ha podido superar el paradigma instrumental en las prácticas de algunos docentes, en contravía al sistema institucional de evaluación y al diseño curricular por competencias. Si bien existe una aproximación al desarrollo por competencias, los instrumentos y estrategias de evaluación no son coherentes con el modelo pedagógico constructivista que tiene la institución.

Igualmente el SIEE, Acuerdo 04 de la institución evidencia estas mismas contradicciones, donde al final establece la promoción a una visión cuantitativa.

La evaluación actual debe ser democrática, participativa, flexible y abierta, como una acción comunicativa que invite al estudiante a través de la autoevaluación y la coevaluación a una reflexión de búsqueda y alternativas para superar las debilidades. De esta manera se advierte el carácter social desde la perspectiva de Bajtin. 


\section{RECOMENDACIONES}

La institución educativa muestra un claro compromiso en su proceso de transformación, el cual se encuentra de pasar de un método tradicional (heteroestructuración), donde la acción preponderante de un agente exterior, a un método activo (autoestructuración), la acción propia del alumno.

Métodos llamados activos (constructivista), basado en un sistema de áreas y por unidades de competencia, esto hace que la institución se encuentre en un proceso de adaptabilidad de sus componentes por eso, presenta en el proceso de evaluación secuelas del método tradicional, pero, también se puede observar desarrollo en la evaluación al nuevo enfoque. La renovación del personal docente también permite una fácil adaptabilidad en su proceso.

Dentro del desarrollo de esta investigación fue claro identificar que la evaluación que se aplica en la institución se presenta en doble vía, entre la evaluación cualitativa y cuantitativa. Esta tendencia permite que la evaluación transite por diferentes rutas.

La conformación de un equipo de gestión académica interdisciplinario, que permita desarrollar cuerpos colegiados encaminados a diseñar e implementar rutas evaluativas académicas coherentes y así mismo el monitoreo permanente de las estrategias e instrumentos de evaluación que estén encaminados a articular las concepciones teóricas de evaluación y sus prácticas.

Ante la ausencia de liderazgo académico entre los directivos, se recomienda el desarrollo de políticas y estrategias para mejorar y perfeccionar los métodos y técnicas de evaluación mediante talleres de capacitación sobre manejo de instrumentos y técnicas de evaluación acorde con el modelo pedagógico. Existe la necesidad de enlazar la gestión directiva con lo pedagógico, de tal manera que el directivo docente no solo lidere procesos innovadores, sino que al interior se haga efectiva la reflexión de comunidad corresponsable en el proceso de evaluación.

En cuanto al SIEE institucional, acuerdo 04 se recomienda hacer los ajustes en lo concerniente a desarrollar las estrategias y procesos de evaluación que apunten a determinar la evaluación en su dimensión cualitativa que ilumine las prácticas de enseñanza para mejorar los procesos de cualificación y medición de manera que sean coherentes con las nuevas realidades y necesidades.

La institución educativa debe integrar dentro del proceso de evaluación a los padres de familia y estudiantes, para establecer espacios de diálogo y compromiso frente a los retos educativos que plantea la institución. Y entender que la evaluación no es el resultado de un proceso final para efectos de promoción sino que la evaluación es un componente más en el proceso de aprendizaje.

Dentro de los objetivos específicos planteados estaba la de analizar las posibles rutas evaluativas que estuvieran en conjunción con el PEI y la evaluación institucional, este objetivo no fue posible desarrollarlo aunque se evidencia como posible ruta, valorar de forma integral los estudiantes lo cual evita ver la evaluación como una práctica limitada a ver resultados finales. De lo anterior es posible evidenciar las tensiones entre la razón formativa y cuantitativa de la evaluación, así mismo, la tensión entre la normatividad del SIEE y la aplicación del Decreto 1290 en lo referente a la evaluación de los aprendizajes o a la promoción. 


\section{REFERENCIAS}

Achury Peñalosa Rodrigo, Rojas Garrido Oscar 2009. La evaluación en el aula, distancia entre la teoría y la praxis, un estudio de caso Escuela Normal Superior San Bernardo Sede Primaria 2009. Maestría en Evaluación. Centro Internacional de Educación y Desarrollo CINDE. Universidad Pedagógica Nacional. Facultad de Educación.

Airasian, P. (2002). La Evaluación en el salón de clases. Biblioteca para la actualización del maestro. México: SEP.

Álvaro Torres Mesías (2010, Noviembre19). Una Reflexión pedagógica sobre la evaluación de los estudiantes para momentos de cambio. Revista Tendencias, pp175, 192.

Bajtin, M Medvedev, P. "La evaluación social, su papel, el enunciado concreto y la construcción poética". Revista Criterios. Número especial. La Habana. 1993 (Trad.Desiderio Navarro).

Casanova, María Antonio. (1997). Manual de evaluación Educativa. Madrid: La Muralla.

Castillo, Santiago. (2003). Compromisos de evaluación Educativa. Madrid. Prentice Hall.

Camilloni, A. Et al. En la evaluación de los aprendizajes en el debate didáctico contemporáneo.Paidós- Buenos Aires- Argentina. Pp.13. DECRETO 1860 de 1994. (Agosto 3 de 1994). Diario Oficial No.41.473, www.mineducación.gov.co/.../articles72061_archivo_pdf_decreto1860_94.pdf DECRETO 1290. (Abril 16 de 2.009). www.mineducación. gov.co/.../articles 187765_archivo_pdf_decreto.

Diccionarios de la Ciencia de la Educación. 1996. Santillana México. Pp. 603.

Documento 11. Ministerio de Educación Nacional. Fundamentación y Orientación para la Implementación del Decreto 1290, 16 de Abril de 2009. Bogotá, Colombia. Pp.105.

García Hoz, Víctor (1981) Educación Personalizada. Madrid: Rialp, 4 edición.

Gardner, Howard (2001) Inteligencias Múltiples: La teoría en la práctica. España. Paidos. Grupo de Trabajo sobre Estándares y Evaluación (en adelante GTEE), liderado por PatriciaArregui del Grupo de Análisis para el Desarrollo (GRADE, Lima, Perú).

Programa de Promoción de la Reforma Educativa en América Latina (PREAL 2008). IA Francesco Giovanni (2010, Noviembre6) Ponencia Evaluación Integral. Foro Nacional de Evaluación. Bogotá, Colombia.

Litwin, E. La evaluación campo de controversias y paradojas o un nuevo lugar para la buena enseñanza. 1998.

Londoño Velásquez Mery, Martínez Alexander, Salazar Norman 2011. Especialista en Evaluación

Pedagógica. Concepciones y prácticas evaluativas en la Institución Educativa

Nuevo Futuro. Universidad Católica de Manizales. Facultad de Educación. 
Maribel Paéz Mendieta (2009, Noviembre 20). Contexto de la Evaluación del 230 al 1290.Magazin IDEP "Aula Urbana". pp.6, 7.

Ministerio de Educación de Colombia: Ley 115 de Febrero de 1994. Por la cual se expide la ley general de educación.

Not Louis (1979). Las Pedagogías del Conocimiento. Fondo de Cultura Económica Ltda. Santa fé de Bogotá.

Ravela, P. 2006. Para comprender las evaluaciones educativas. PREAL. Santiago de Chile.

\section{CINDE.}

Ravela, P. 2008. ¿Debemos creerles a las evaluaciones estandarizadas?. PREAL. Santiago de Chile. CINDE.

Rul Gargallo, J. (1994). La evaluación comunicativa. Barcelona, Generalitat de Cataluya.

Rotavista Valderrama Andrea del Pilar, Talero Edgar Alberto. Maestría en Pedagogía. La

Evaluación como práctica reflexiva: un medio para comprender y mejorar la enseñanza.

Universidad de la Sabana. Facultad de Educación.

Sacristán, J y Pérez, A. P (2000). Comprender y Transformar la enseñanza (9 ed). Madrid. Morata.

Santos Guerra, Miguel. (1996). Un enfoque práctico de la evaluación de los alumnos, profesores y centros educativos y materiales didácticos. Buenos Aires. Magisterio del Río de la Plata.

Santibáñez Riquelme, Juan. (2001). Manual para la evaluación del aprendizaje estudiantil conceptos, procedimientos, análisis e interpretación para el proceso educativo. México. Trillas.

Shepard, L. 2008. La evaluación en el aula. México. Publicación INEE. Traducción del Capítulo 17 del Libro Educational Measurement, 4a. Edition, 2006. USA, Robert Brennan Editor.

TYLER, R . Diversas Concepciones Sobre Evaluación.

https://www.google.com.co/search?q=Tyler+concepciones+sobre+evaluaci\%C3

\%B3n\&oq=Tyler+concepciones+sobre+evaluaci\%C3\%B3n\&aqs=chrome..69i57.

134464j0j7\&sourceid=chrome\&espv $=210 \&$ es_sm $=93 \&$ ie $=$ UTF-8 